\title{
Evaluación prospectiva de la venografía mediante angioTC en el diagnóstico de enfermedad tromboembólica
}

\author{
MAX ANDRESEN H. ${ }^{1}$, ALEJANDRO GONZÁLEZ ${ }^{2}$, ORLANDO DÍAZ P. ${ }^{1}$, \\ LUIS MENESES ${ }^{3}$, MARIO FAVA $^{3}$, ELISA ORLANDINI ${ }^{a}$, LETICIA CLEDE ${ }^{a}$, \\ TOMÁS REGUEIRA H. ${ }^{1}$, RICARDO CASTRO ${ }^{1}$
}

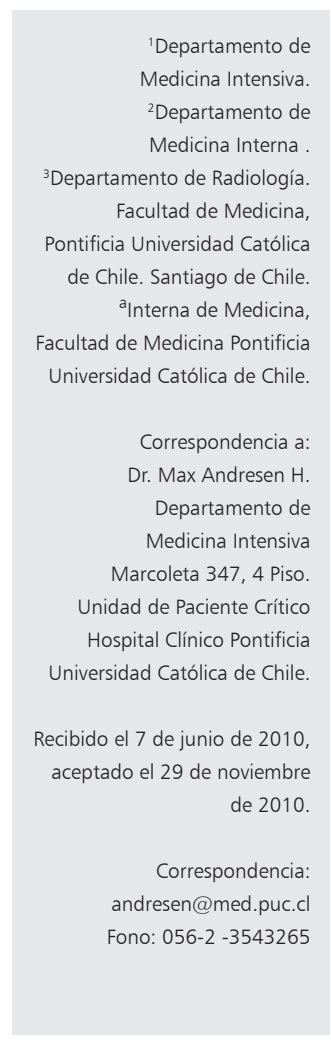

\section{Diagnostic yield of CT pulmonary angiography and venography for thromboembolic disease}

Background: CT pulmonary angiography is the diagnostic procedure of choice for non-massive pulmonary embolism. Aim: To assess the diagnostic yield for thromboembolic disease of CT pulmonary angiography and venography using a 64- slice multidetector tomography. Material and Methods: Prospective study of patients with a clinical suspicion of thromboembolic disease, subjected to CT pulmonary angiography and venography. The presence and location of pulmonary thromboembolism, of isolated or concomitant deep venous thrombosis and of other significant radiological findings, were registered. Results: A 64-MDCT scanner was performed to 893 patients and thromboembolic disease was demonstrated in 240. Pulmonary thromboembolism was diagnosed in 218 patients. It was concomitant with deep venous thrombosis in 79 patients (36\%) and isolated in the rest. Thirty five of the 218 patients with pulmonary thromboembolism had radiological evidence of right ventricular overload. Twenty two patients (10\%) had an isolated deep venous thrombosis. In 65 patients with pulmonary thromboembolism (30\%) a possibly new or old malignant lesion, was observed. Seventy one of 653 patients without evidence of thromboembolic disease had potentially pathological findings on CT. Conclusions: The combined use of CT pulmonary arteriography and venography using a 64 MDCT scanner increases the diagnostic yield of the procedure for thromboembolic disease. It also allows the diagnosis of other related conditions, specially malignant tumors.

(Rev Med Chile 2010; 138: 1480-1486).

Key words: Angiography; Thromboembolism; Tomography scanners, $x$-Ray computed.
L a angiografía pulmonar por tomografía computada (angioTC) es el examen de elección en el proceso diagnóstico del tromboembolismo pulmonar (TEP) no masivo ${ }^{1}$. La trombosis venosa profunda (TVP) y el TEP deben considerarse parte del mismo proceso patológico, que es la enfermedad tromboembólica (ETE), y por lo tanto, evaluadas en conjunto ${ }^{2}$. Así, distintos autores incluyen el estudio de extremidades inferiores y de vasos pulmonares en los distintos algoritmos ${ }^{3}$, conociendo además el elevado riesgo de muerte derivado de una embolia recurrente proveniente de trombosis venosa profunda de extremidades inferiores o de pelvis ${ }^{4}$.

Para el estudio del sistema venoso de las extremidades inferiores se dispone de variados métodos de apoyo diagnóstico, como la venografía con contraste, la ecografía más Doppler y la cintigrafía, entre otras. Sin embargo, el uso combinado de angioTC y venografía por TC (venoTC) simplifica 
y acorta la evaluación de la ETE, ya que evalúa el estado de las venas de las extremidades inferiores, fuente frecuente de origen del TEP, y no requiere el uso de medio de contraste adicional ${ }^{2}$. Así, estos métodos permiten diagnosticar simultáneamente el TEP y la TVP. El examen venoso complementario se realiza cuando el material de contraste inyectado para la angiografía pulmonar circula a través del sistema venoso profundo de las extremidades inferiores. De esta forma, no sólo permite conocer el origen más probable del TEP sino que también podría incrementar los diagnósticos de ETE si detecta pacientes con trombosis venosa en ausencia de $\mathrm{TEP}^{5}$. Existen otras ventajas potenciales de este tipo de estudio. Una de ellas es la determinación de la masa del coágulo, información que puede ayudar a definir terapias específicas, pues estas dependen de la magnitud radiológica de la embolia, la ubicación y tamaño de los trombos en el territorio venoso y la repercusión tomográfica de la embolia sobre las cavidades derechas. Otra ventaja es que permite el hallazgo de otras patologías, relacionadas o no con el origen de la ETE como, por ejemplo, tumores 5 .

El principal objetivo del presente trabajo fue evaluar nuestra experiencia con los resultados del uso combinado de angioTC empleando un tomógrafo multidetector de 64 canales (64-MDCT) y venoTC en el diagnóstico de la ETE. Un objetivo secundario ha sido describir los hallazgos concomitantes de potencial utilidad clínica.

\section{Pacientes y Métodos}

Estudio prospectivo realizado en el Hospital Clínico de la Pontificia Universidad Católica de Chile entre enero de 2007 y junio de 2009, en pacientes adultos con sospecha de TEP. A todos se les practicó angioTC y, complementariamente, una venografía por TC. Los criterios de exclusión fueron: alergia severa a agentes de contraste yodado, alteración de la función renal (creatinina plasmática mayor de $1,5 \mathrm{mg} / \mathrm{dl}$ ), embarazo y edad menor de 18 años. Nuestro protocolo radiológico corresponde al estándar empleado en los estudios clínicos rutinarios, sin modificaciones.

Técnica radiológica². Todos los estudios fueron realizados con un tomógrafo multidetector 64MDCT (Light Speed, General Electric, EE.UU.).
Las imágenes fueron adquiridas en dirección caudo-craneana desde el nivel del diafragma hasta los ápices pulmonares en una sola contención de la respiración. Los exámenes fueron adquiridos con $120 \mathrm{kV}, 100 \mathrm{mAs}$; colimación de $64 \times 0,625$; tiempo de rotación de 0,4 s y pitch de 1,172 ; lo que determina un tiempo de adquisición de aproximadamente $5 \mathrm{~s}$. A los pacientes se les administró $70 \mathrm{ml}$ de contraste yodado no-iónico iv (Optiray 320, Tyco Healthcare, Mallinkrodt, Hazelwood, MO, USA) a un flujo $5 \mathrm{ml} / \mathrm{seg}$, seguido de $50 \mathrm{ml}$ de solución salina isotónica (bolus chaser) a través de teflón $18 \mathrm{G}$ ubicado en vena cubital o acceso venoso disponible. Se utilizó técnica de bolus tacking colocando Región de Interés en el tronco de la arteria pulmonar. El umbral para iniciar la adquisición fue de 100 U.H. con un retraso de 8 segundos luego de alcanzado el umbral. El estudio se complementó con imágenes obtenidas tardíamente, a los 3 min, de abdomen, pelvis y extremidades hasta las fosas poplíteas, como estudio venográfico para detectar trombosis venosa profunda. Todas las imágenes fueron analizadas como cortes axiales finos y los "reformateos multiplanares" y de Proyección de Máxima Intensidad fueron utilizados a discreción del radiólogo que evaluaba el estudio. Del punto de vista cardiaco se describió la presencia de signos de sobrecarga derecha, dados por alteración en la relación ventrículo derecho/ventrículo izquierdo. Además, se realizó una descripción de abdomen y pelvis en búsqueda de patología incidental, particularmente masas sospechosas de neoplasia, lo cual se describió en todos los informes radiológicos de los angioTC y las venoTC solicitados por sospecha de TEP. Esta última descripción se incluyó en un análisis retrospectivo de todos los informes radiológicos de angioTC con venografía concomitante, en la búsqueda de hallazgos incidentales de nuevas patologías que pudieran estar relacionadas o no a la enfermedad tromboembólica.

Análisis estadístico. Para calcular el tamaño de la muestra hemos asumido que aproximadamente $30 \%$ de las angioTC y venoTC solicitadas por sospecha de ETE en una población general demostrarán la enfermedad. Según esto, entre 520 y 780 pacientes permitirían detectar un aumento del rendimiento diagnóstico de 2,5 a 3\% al emplear ambos exámenes concomitantemente en lugar de la angioTC aislada. Se ha considerado una potencia de $90 \%$ y un nivel de significación de $5 \%$, 
asumiendo una proporción de pares discordantes de $5 \%$ y como método de análisis una prueba de McNemar para proporciones pareadas. Para comparar proporciones no pareadas se empleó prueba de chi cuadrado. En todos los casos se consideró significativo un valor de $\mathrm{p}<0,05$.

\section{Resultados}

Durante el período estudiado se realizó angioTC y venoTC a 893 pacientes, demostrándose ETE en 240. Como se describe en la Tabla y Figura 1 , estos casos correspondieron a TEP en 218 pacientes: TEP aislado en $139(63,7 \%)$ y concomitante con TVP en 79 pacientes $(36,2 \%)$. A su vez, 22 pacientes presentaron TVP aislada (10\%).

Tromboembolismo pulmonar. En los 218 pacientes con TEP, la Tabla 1 indica que el compromiso de ramas segmentarias de la arteria pulmonar fue el más frecuente $(33,9 \%)$, seguido por el compromiso lobar (22\%), de tronco y arterias principales $(19 \%)$ y de ramas subsegmentarias $(10,5 \%)$.

Trombosis venosa profunda. Se demostró TVP en 101 pacientes, 79 de los cuales tenía concomitantemente un TEP y 22 poseía una trombosis aislada. La trombosis afectó más fre- cuente a las venas femorales e ilíacas (Tabla 1). Esta distribución no fue diferente al comparar los pacientes con TVP aislada y aquellos con TVP concomitante con TEP.

\section{Tabla 1. Características clínicas y demográficas de $\mathbf{2 4 0}$ pacientes con enfermedad tromboembólica}

\begin{tabular}{|lc|}
\hline Edad, años & $58 \pm \mathrm{DE}$ \\
\hline Sexo, Hombres/Mujeres & $117 / 101$ \\
\hline Enfermedad tromboembólica, $\mathrm{n}$ & 240 \\
TEP aislado & 139 \\
TEP + TVP & 79 \\
TVP aislada & 22 \\
\hline Localización del TEP, $\mathrm{n}(\%)$ & 218 \\
Tronco y arterias principales & $42(19,2)$ \\
Lobar & $48(22)$ \\
Multisegmentario & $31(14,2)$ \\
Segmentario & $74(33,9)$ \\
Subsegmentario & $23(10,6)$ \\
Localización de la TVP, $\mathrm{n}(\%)$ & 101 \\
Venas femorales & $34(33,6)$ \\
Venas ilíacas & $30(29,7)$ \\
Venas poplíteas & $13(12,8)$ \\
\hline
\end{tabular}

Los resultados se expresan como promedios $\pm 1 \mathrm{DE}$ (desviación estándar) o números (porcentajes), según corresponda. TEP: Tromboembolismo pulmonar; TVP: trombosis venosa profunda.

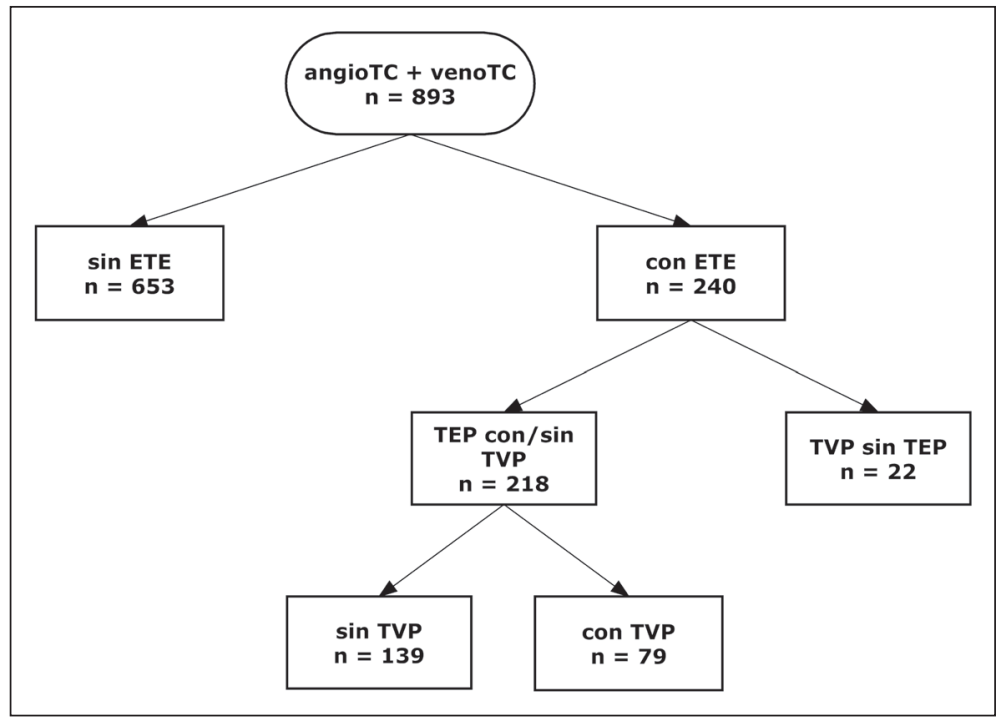

Figura 1. Resultados de la angiografía pulmonar y venografía por tomografía computada en 893 pacientes con sospecha de enfermedad tromboembólica. 
Venografía y angioTC en enfermedad tromboembólica - M. Andresen et al

Rendimiento diagnóstico. La Tabla 2 muestra que en los 893 pacientes estudiados por sospecha de ETE, el diagnóstico se confirmó en 218 casos por la presencia de TEP $(24,4 \%)$ y en otros 22 casos $(2,5 \%)$ por la presencia de TVP aislada en la venoTC. En otras palabras, el estudio del sistema venoso profundo aumentó significativamente el rendimiento diagnóstico de la angioTC en 2,46\% (prueba de McNemar; $\mathrm{p}<0,05$ ).

Sobrecarga ventricular derecha. Se observó evidencia radiográfica de sobrecarga ventricular derecha en 35 de los 218 pacientes con TEP $(16,0 \%)$ (Tabla 3$)$. En estos pacientes, la sobrecarga ventricular fue significativamente más frecuente cuando existió concomitantemente un TEP y una TVP que cuando la TVP se presentó aisladamente $(30,3 \%$ versus $7,9 \%$, respectivamente; $\mathrm{p}<0,05)$.

Otras lesiones. En 65 de los 218 pacientes con TEP (30\%), se observó una lesión de aspecto neoplásico, nueva o antigua. De estas lesiones sospechosas de neoplasia, 79\% tenía carácter sólido y $15 \%$ presentaba características propias de lesiones linfoproliferativas. Entre las lesiones sólidas, el cáncer de pulmón fue el más frecuente y correspondió a $23 \%$ de todas las neoplasias. El $6 \%$ de las lesiones tenía aspecto benigno. En los 653 casos negativos para ETE, 71 exámenes (11\%)
Tabla 2. Comparación del rendimiento diagnóstico en la enfermedad tromboembólica al emplear angiografía pulmonar por tomografía computada aislada o acompañada de venografía por tomografía computada

\begin{tabular}{|llrrr|}
\hline & & \multicolumn{2}{c}{ ETE por } & Total \\
& & \multicolumn{2}{c}{ angioTC } & \\
& & \multicolumn{1}{c}{ Sí } & No & \\
\hline ETE por angioTC & Sí & 218 & 22 & 240 \\
+ venoTC & No & 0 & 653 & 653 \\
& Total & 218 & 675 & 893 \\
\hline
\end{tabular}

ETE: enfermedad tromboembólica; angioTC: angiografía pulmonar por tomografía computada; venoTC: venografía por tomografía computada.

Tabla 3. Sobrecarga ventricular derecha en pacientes con tromboembolismo pulmonar con y sin trombosis venosa profunda concomitante

\begin{tabular}{|c|c|c|c|c|}
\hline & & Sobre & tricular & Total \\
\hline & & Sí & No & \\
\hline & Sí & 24 & 11 & 79 \\
\hline IVT & No & 11 & 128 & 139 \\
\hline Total & & 35 & 139 & 218 \\
\hline
\end{tabular}

TVP: trombosis venosa profunda.

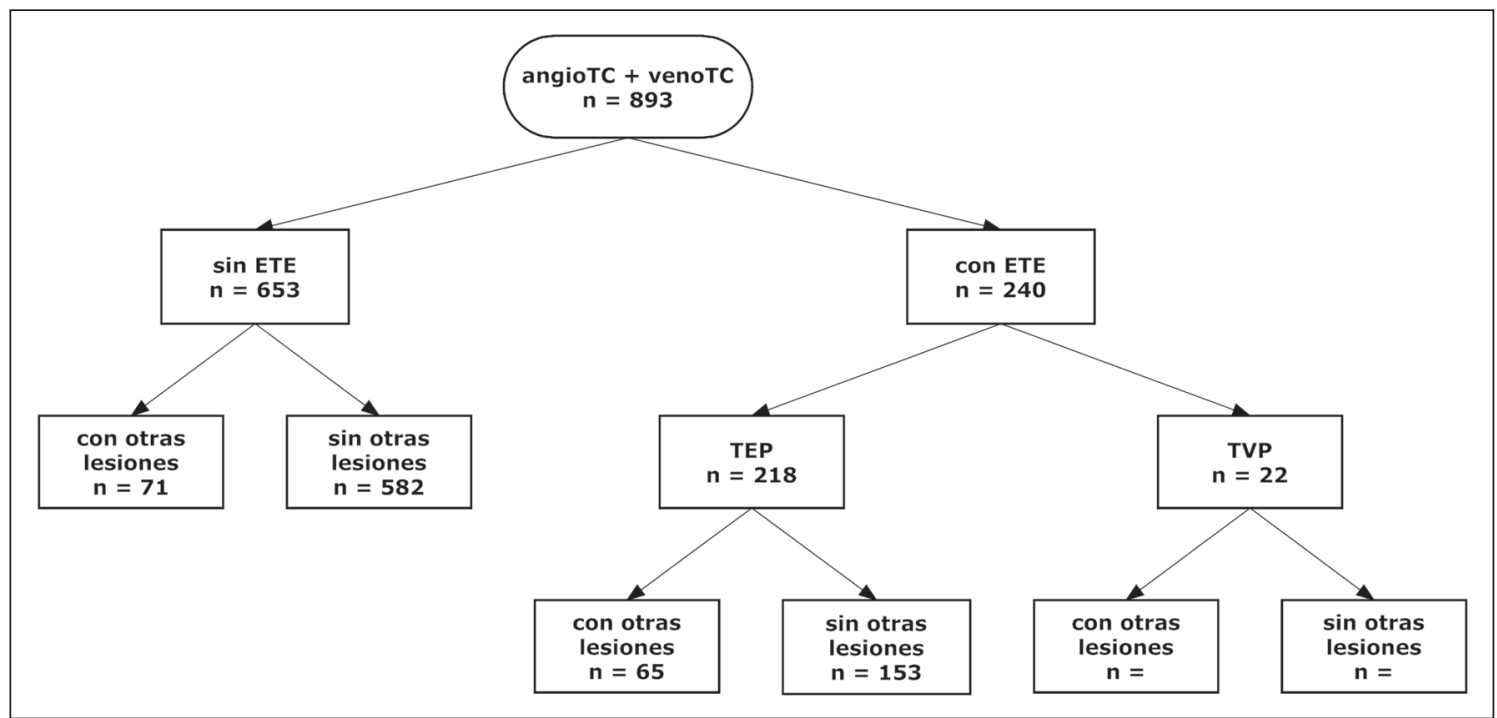

Figura 2. Frecuencia de diagnósticos alternativos en la angiografía pulmonar y venografía por tomografía computada en 893 pacientes con sospecha de enfermedad tromboembólica. 
presentaron hallazgos patológicos significativos. En 53 casos (75\%) estos también correspondían a lesiones de aspecto neoplásico, principalmente de carácter sólido (83\%). El aspecto radiográfico de cáncer de pulmón nuevamente fue el más frecuente, correspondiendo a $23 \%$ de todas las neoplasias.

\section{Discusión}

Actualmente, sabemos que el diagnóstico y tratamiento precoz de la ETE mejora considerablemente la sobrevida ${ }^{6}$. La angioTC es usada frecuentemente en la evaluación del TEP ya que muestra con precisión émbolos centrales, segmentarios y subsegmentarios y proporciona, además, imágenes detalladas del tórax que pueden proporcionar diagnósticos alternativos para los síntomas respiratorios de un paciente. Así el British Thoracic Society Standards of Care Committee Pulmonary Embolism Development Group $p^{7}$ recomienda la angioTC como el método de diagnóstico inicial en el TEP no masivo, como también lo es para la Sociedad Europea de Cardiología en sus guías clínicas de manejo del TEP ${ }^{8}$.

Por su parte, la angioTC complementada con la venografía por TC busca el tromboembolismo como una enfermedad, mezclando el examen de la fuente (venas) y el objetivo (el árbol pulmonar arterial) en un estudio único y rápido, 10-12.

En la actualidad, la venoTC se está haciendo más popular como medio de diagnóstico complementario y esto hace necesario no sólo definir su eficacia, sino también sus posibles efectos adversos y otros posibles beneficios. Aún no existe consenso de cuál sea la técnica ideal, ya que cuando se la compara con el ultrasonido con Doppler más compresión, son equivalentes, logrando este último una sensibilidad y especificidad aproximada de $95 \%$ para las venas femorales y poplíteas ${ }^{12-14}$.

En la literatura se estima que más de $90 \%$ de los émbolos pulmonares provienen de las extremidades inferiores ${ }^{6}$. En nuestra serie, la existencia de un émbolo pulmonar estuvo acompañada por una TVP concomitante en $30 \%$ de los casos, lo que permite especular que en el $70 \%$ restante hubo desprendimiento total del trombo responsable del TEP. Esta especulación se basa en que la venoTC permite evaluar también el sistema venoso pelviano, vena cava inferior y las cavidades cardiacas derechas, por lo que un resultado negativo hace muy improbable la posibilidad de otros orígenes.

La incidencia de TVP sin TEP concomitante oscila entre $0,2 \%$ y $5 \%$ en pacientes con sospecha de embolia pulmonar en que el diagnóstico se ha efectuado empleando venografía por $\mathrm{TC}^{15,16}$. En términos prácticos, esto significa que la venoTC puede aumentar el rendimiento diagnóstico de la ETE entre $2,4 \%$ y $28 \%{ }^{17,18}$. Este amplio rango probablemente se explique por los variados tomógrafos utilizados en estos estudios ${ }^{19}$. Nuestros resultados, empleando un tomógrafo multidetector 64-MDCT, apoyan el hecho que la venografía por TC aumenta el rendimiento de la angioTC en el diagnóstico de ETE ya que permitió detec$\operatorname{tar} 22$ casos de TVP sin TEP que requirieron el mismo tratamiento anticoagulante. Así, el rendimiento diagnóstico aumentó 2,46\%. Este mayor rendimiento fue estadísticamente significativo, pero a primera vista numéricamente marginal en comparación con otros estudios previos. Es conveniente recordar, sin embargo, que la ETE no diagnosticada y consecuentemente no tratada es potencialmente mortal por lo que beneficios aparentemente menores son igualmente relevantes desde un punto de vista clínico. Es posible que la menor utilidad de la venografía por TC del presente estudio se deba a que el tomógrafo multidetector de 64 canales es más rápido y disminuye el grosor de los cortes en comparación con modelos más antiguos ${ }^{17}$. Estas características permiten visualizar mejor los vasos pulmonares y detectar un número considerable de TEP subsegmentarios $(10,4 \%)$ que de otra manera no habrían sido percibidos. En consecuencia, aquellos estudios que no han contado con la tecnología multidetector disminuyen su capacidad para diagnosticar TEP de ramas menores ${ }^{20}$ lo que necesariamente aumenta el rendimiento de la venografía por TC.

Al no existir una evaluación de la probabilidad pretest en el presente estudio, no podemos establecer si la venografía podría ser de mayor utilidad en ciertos grupos de riesgo. Cabe destacar, en todo caso, que el mayor rendimiento fue independiente de tal análisis de riesgo.

Una de las principales consideraciones negativas de agregar la venoTC a la angiografía pulmonar por TC, es que aumenta la dosis de radiación. Se ha encontrado evidencia que agregar venografía por TC aumenta la dosis de radiación gonadal y de pelvis en comparación con la angioTC aisla$\mathrm{da}^{21}$. Sin embargo, no hay evidencia de que estas 
Venografía y angioTC en enfermedad tromboembólica - M. Andresen et al

dosis sean deletéreas, ya que se encontrarían por debajo de los umbrales peligrosos comunicados por la Comisión Internacional sobre la Protección Radiológica ${ }^{21}$. Algunos investigadores han sugerido emplear secciones discontinuas transversales, con mayor espacio entre cada sección. Con este tipo de protocolo se ha observado una reducción de la dosis integral de hasta $80 \%$. Sin embargo, la especificidad del examen disminuye debido a una mayor dificultad en la interpretación radiológica ${ }^{22}$, lo que se debe a que las secciones discontinuas deterioran la interpretación en venas iliacas y poplíteas y aumentan los artefactos que imitan trombos. También, en la línea de reducción de la dosis de radiación se ha sugerido la realización del venoTC circunscrito a las venas femorales y poplíteas ${ }^{3}$.

La solicitud más selectiva de la venoTC, que considerara, por ejemplo, los grados de sospecha clínica, el perfil de riesgo y los resultados de biomarcadores como el dímero D o el BNP, podría constituir una estrategia adecuada para atenuar los posibles riesgos y los mayores costos. El presente trabajo no permite efectuar un análisis detallado de la población más beneficiada por un estudio combinado.

Respecto de la gravedad de la embolia pulmonar, nuestros datos indican que $16 \%$ presentaba repercusión ventricular derecha en la tomografía. De ellos, $68,5 \%$ tenía un foco embolígeno concomitante $y$, por ende, el riesgo de una nueva embolia (Tabla 3). La falla derecha aguda se puede determinar en la angioTC pulmonar midiendo las dimensiones de las cavidades derechas del corazón o de las estructuras venosas tales como la vena cava superior o la vena azigos, que son elementos demostrados como predictores de mal pronóstico en el TEP ${ }^{23}$.

\section{Hallazgos complementarios}

Respecto de los diagnósticos adicionales, en nuestro estudio la presencia de neoplasia asociada a enfermedad tromboembólica fue cercana al 30\%, mayor que los datos estimados en la literatura mundial $(8-17 \%)^{3}$. Esto se explicaría por la adición de la venografía, que permite una evaluación más extensa al incluir abdomen y pelvis, posibilitando la detección de otro tipo de lesiones y agregando posibles diagnósticos de patologías que modificarían el pronóstico único de una embolia pulmonar $^{24}$.
En conclusión, nuestro estudio muestra que el uso combinado de angiografía pulmonar por TC y venografía por TC empleando un tomógrafo multidetector de 64 canales aumenta el rendimiento diagnóstico de la enfermedad tromboembólica, como también de su localización y repercusión. Además permite diagnosticar otras patologías concomitantes, en general de naturaleza neoplásica, relacionadas o no con la presencia de ETE.

\section{Referencias}

1. Perrier A, Howarth N, Didier D, Loubeyre P, Unger PF, de Moerloose P, et al. Performance of Helical Computed Tomography in Unselected Outpatients with Suspected Pulmonary Embolism. Ann Intern Med 2001; 135: 8897.

2. Loud P, Katz D, Klippenstein D, Shah R, Grossman Z. Combined CT Venography and Pulmonary Angiography in Suspected Thromboembolic Disease: Diagnostic Accuracy for Deep Venous Evaluation. AJR 2000; 174: 61-5.

3. Stein P, Woodard P, Weg J, Wakefield T, Tapson V, Sostman H, et al. PIOPED II Investigators. Diagnostic pathways in acute pulmonary embolism: recommendations of the PIOPED II Investigators. Radiology 2007; 242: 15-21.

4. Moser K. Venous thromboembolism. Am Rev Respir Dis 1990; 141: 235-49.

5. Bierry G, Holl G, Kellner F, Riehm S, Roedlich MN, Greget M, et al. Venous Thromboembolism and Occult Malignancy: Simultaneous Detection During Pulmonary CT Angiography with CT Venography. AJR 2008; 191: 885-9.

6. Tapson VF. Acute pulmonary embolism. N Engl J Med 2008; 358: 1037-52.

7. British Thoracic Society Standards of Care Committee Pulmonary Embolism Guideline Development Group. British Thoracic Society guidelines for the management of suspected acute pulmonary embolism. Thorax 2003; 58: 470-83.

8. Torbicki A, Perrier A, Konstantinides S, Agnelli G, Galie N, Pruszczyk P, et al. Guidelines on the diagnosis and management of acute pulmonary embolism: the Task Force for the Diagnosis and Management of Acute Pulmonary Embolism of the European Society of Cardiology (ESC). Eur Heart J 2008; 29: 2276-315.

9. Ghaye B, Szapiro D, Willems V. Combined CT venography of the lower limbs and spiral CT angiography of pulmonary arteries in acute pulmonary embolism: 
preliminary results of a prospective study. JBR-BTR 2000; 83: 271-8.

10. Garg K, Kemp JL, Wojeik D. Thromboembolic disease: comparison of combined CT pulmonary angiography and venography with bilateral leg sonography in 70 patients. Am J Roentgenol 2000; 175: 997-1001.

11. Coche EE, Hamoir XL, Hammer FD. Using dual-detector helical CT angiography to detect deep venous thrombosis in patients with suspicion of pulmonary embolism: diagnostic value and additional findings. Am J Roentgenol 2201; 176: 1035-9.

12. Cham MD, Yankelevitz DF, Shaham D. Deep venous thrombosis: detection by using indirect CT venography, Radiology 2000; 216: 744-51.

13. Cogo A, Lensing A, Prandoni P. Comparison of real-time B-mode ultrasonography and doppler ultrasound with contrast venography in the diagnosis of venous thrombosis in symptomatic outpatients. Thromb Haemost 1993; 70: 404-7.

14. Davidson B, Elliot C, Lensing A. Low accuracy of color Doppler ultrasound in the detection of proximal leg vein thrombosis in asymptomatic high-risk patients: the RD heparin Arthroplasty Group. Ann Intern Med 1992; 117: 735-8.

15. Cham M, Yankelevitz D, Henschke C. Thromboembolic Disease Detection at Indirect CT Venography versus CT Pulmonary Angiography. Radiology 2005; 234: 591-4.

16. Ciccotosto C, Goodman L, Washington L, Quiroz F. Indirect CT venography following CT pulmonary angiography. J Thorac Imaging 2002; 17: 18-27.

17. Yasui T, Tanabe N, Terada J. Multidetector-Row Com- puted Tomography Management of Acute Pulmonary Embolism. Circ J 2007; 71: 1948-54.

18. Cham M, Yankelevitz D, Shaham D, Shah A. Deep Venous Thrombosis: Detection by Using Indirect CT Venography. Radiology 2000; 216: 744-51.

19. Prologo J, Gilkeson R, Díaz M, Cummings M. The Effect of Single-Detector CT Versus MDCT on Clinical Outcomes in Patients with Suspected Acute Pulmonary Embolism and Negative Results on CT Pulmonary Angiography. AJR 2005; 184: 1231-5.

20. Douma R, Hofstee H, Schaefer-Prokop C, van Waesberghe J, Lely R, Kamphuisen P, et al. Comparison of 4- and 64-slice CT scanning in the diagnosis of pulmonary embolism. Thromb Haemost 2010; 103: 242-6.

21. Rademaker J, Griesshaber V, Hidajat N. Combined CT pulmonary angiography and venography for diagnosis of pulmonary embolism and deep venous thrombosis: radiation dose. J Thorac Imaging 2001; 16: 297-9.

22. Hunsaker A, Zou K, Poh A, Trotman-Dickenson B, Jacobson F, Gill R, et al. Routine Pelvic and Lower Extremity CT Venography in Patients Undergoing Pulmonary CT Angiography. AJR 2008; 190: 322-6.

23. Ghuysen A, Ghaye B, Willems V, Lambermont B, Gerard P, Dondelinger RF, et al. Computed tomographic pulmonary angiography and prognostic significance in patients with acute pulmonary embolism. Thorax 2005; 60: 956-61.

24. Carrier M, Le Gal G, Wells P, Fergusson D, Ramsay T, Rodger M, Systematic Review: The Trousseau Syndrome Revisited: Should We Screen Extensively for Cancer in Patients with Venous Thromboembolism?. Ann Intern Med 2008; 149: 323-33. 